\title{
Caractérisation zootechnique des ovins de race Sardi au Maroc
}

\author{
A. Chikhi ${ }^{1}$ I. Boujenane ${ }^{2 *}$
}

\begin{abstract}
Mots-clés
Ovin Sardi - Performance animale Performance de reproduction - Poids corporel - Laine - Carcasse - Maroc.
\end{abstract}

Résumé

\begin{abstract}
Un total de 811 performances de reproduction, 1430 poids de toison, 1024 performances de croissance et de viabilité, et 154 performances d'engraissement et d'abattage des ovins de race Sardi ont été analysées. Ces performances ont été collectées durant sept campagnes allant de 1993-1994 à 1999-2000 au domaine expérimental de Déroua à l'Institut national de la recherche agronomique. L'analyse des données a montré que les brebis avaient une durée de la gravidité de 151 jours, un taux de fertilité de 98 p. 100, des tailles de portée à la naissance et au sevrage (90 jours) respectivement de 1,29 et 1,25, et des poids de portée à la naissance et au sevrage respectivement de 5,30 et $27,9 \mathrm{~kg}$. Les agneaux ont pesé 4,10 kg à la naissance, $10,9 \mathrm{~kg}$ à 30 jours et $22,5 \mathrm{~kg}$ à 90 jours. La vitesse de croissance moyenne a été de $224 \mathrm{~g} / \mathrm{j}$ de la naissance à 30 jours et de $194 \mathrm{~g} / \mathrm{j}$ entre 30 et 90 jours. Le taux de mortalité entre la naissance et 90 jours des agneaux a été de 7 p. 100. Après un engraissement postsevrage d'une durée moyenne de 66,4 jours, les agneaux nés simples ont réalisé un gain de poids moyen quotidien de $283 \mathrm{~g} / \mathrm{j}$ et un indice de consommation de 4,87 $\mathrm{kg}$ d'aliment $/ \mathrm{kg}$ de gain de poids. Abattus à l'âge moyen de 168 jours, ils ont réalisé un poids vif à l'abattage de $45,5 \mathrm{~kg}$, un poids de carcasse chaude de $23,2 \mathrm{~kg}$, un rendement économique de 50,9 p. 100 et un poids du gras de rognon de $295 \mathrm{~g}$. II a été conclu que la race Sardi a de très bonnes potentialités qu'il est possible d'exploiter pour l'augmentation de la production de viande au Maroc.
\end{abstract}

\section{INTRODUCTION}

La caractérisation des ressources génétiques locales est une étape nécessaire pour déterminer la méthode génétique appropriée pour leur amélioration. La race Sardi est parmi les principales races ovines locales marocaines (1). Cependant, les travaux entrepris sur cette race ont été souvent réalisés dans des conditions qui ne lui permettent pas d'extérioriser ses potentialités réelles $(3,8,11)$. Par conséquent, de grands efforts doivent être déployés pour faire une mise au point sur les vraies potentialités de cette race et évaluer les marges et les voies de l'amélioration de sa productivité. Le but de cette étude a été d'évaluer les performances de reproduction des brebis et de production des agneaux dans des conditions intensives pour avoir une bonne appréciation du niveau de performances des ovins de race Sardi.

1. Centre régional de la recherche agronomique de Tadla, Institut national de la recherche agronomique,

BP 567, Béni Mellal 23000, Maroc

Tél./fax: 21223440006 ; e-mail: chikhi@awamia.inra.org.ma

2. Département des productions animales,

Institut agronomique et vétérinaire Hassan II,

BP 6202, Rabat-Instituts, 10101 Rabat, Maroc

Tél./fax: 21237776420 ; e-mail: i.boujenane@iav.ac.ma

* Auteur pour la correspondance

\section{MATERIEL ET METHODES}

\section{Origine des données}

L'étude a eu lieu dans le domaine expérimental de Déroua (DED) à l'Institut national de la recherche agronomique (Inra) de Béni Mellal, localisé à $300 \mathrm{~km}$ au sud-est de Rabat. La pluviométrie annuelle moyenne est de $386 \mathrm{~mm}$, la température oscille entre $-4{ }^{\circ} \mathrm{C}$ au mois de janvier et $45^{\circ} \mathrm{C}$ au mois de juillet, avec une moyenne de $18{ }^{\circ} \mathrm{C}$.

\section{Distribution géographique et description phénotypique}

La race Sardi appartient à la population ovine des plateaux de l'ouest du Maroc. Elle peuple les régions situées dans le triangle formé par Béni Mellal, Settat et Khouribga. Son effectif s'élève à presque 2,1 millions de têtes en 1996, soit 12,9 p. 100 de l'effectif national ovin (1).

Elle est caractérisée par une tête blanche avec un museau noir, des tâches noires autour des yeux, ce qui lui vaut l'appellation de « race à lunettes ». Chez le mâle, le profil est busqué, le crâne est large et porte des cornes blanches et puissantes. Chez la femelle, le chanfrein est presque droit et la tête est dépourvue de cornes. Les pattes sont blanches et sans laine, avec des points noirs aux extrémités et à la pointe des jarrets. Les ovins de race Sardi ont une grande taille, 
variant de 0,80 à $0,90 \mathrm{~m}$ chez le mâle et de 0,55 à $0,65 \mathrm{~m}$ chez la femelle. Le poids à l'âge adulte varie de 70 à $90 \mathrm{~kg}$ chez le mâle et de 45 à $55 \mathrm{~kg}$ chez la femelle. La toison est blanche et pèse en moyenne $1,5 \mathrm{~kg}$ chez la femelle et $2,5 \mathrm{~kg}$ chez le mâle (1).

\section{Conduite des animaux}

Les brebis ont été soumises à une lutte contrôlée nocturne d'une durée de 60 jours pendant les mois de juin et juillet. Les lots de lutte ont été constitués d'un bélier et de 20 à 24 brebis. Les béliers, préalablement peints sur leur partie abdominale afin d'identifier les brebis saillies, ont été introduits à $17 \mathrm{~h}$ et retirés à $8 \mathrm{~h}$ du lendemain. A la fin de la période de la lutte, toutes les brebis ont été regroupées en un seul troupeau.

Les animaux ont été conduits en stabulation permanente. L'alimentation a été à base du foin de luzerne, de paille, d'orge grain, de maïs grain, de pulpe sèche de betterave (PSB), de tourteau de tournesol (TT) et d'un complément minéral vitaminé (CMV). Les quantités distribuées ont varié en fonction des besoins physiologiques des animaux. Pendant la période de lutte, les brebis on reçu une complémentation de 250 à $300 \mathrm{~g}$ d'orge, 80 à $100 \mathrm{~g}$ de TT et $10 \mathrm{~g}$ de CMV. En fin de gravidité, les aliments complémentaires ont été $400 \mathrm{~g}$ d'orge, 100 à $120 \mathrm{~g}$ de TT et 10 à $20 \mathrm{~g}$ de CMV. Durant la période de lactation, la ration alimentaire a été identique à celle de fin de gravidité augmentée de $300 \mathrm{~g}$ de PSB.

L'alimentation des agneaux a été exclusivement à base du lait maternel au cours du premier mois de leur vie. A partir du deuxième mois, les agneaux ont reçu un concentré spécifique composé de 58 p. 100 d'orge, 40 p. 100 de TT et 2 p. 100 de CMV.

Au sevrage vers l'âge de 90 jours, les agneaux et les agnelles de renouvellement ont été sélectionnés et conduits selon un rythme normal pour être aptes à la reproduction à l'âge de 18 mois. Une trentaine de mâles nés simples, parmi les non retenus pour le renouvellement, ont été engraissés chaque année pendant une durée de 60 à 70 jours, puis abattus. Les agneaux mis à l'engraissement ont été répartis en lots de six à sept agneaux. L'engraissement proprement dit a commencé après une dizaine de jours d'adaptation. L'âge moyen des agneaux au début de l'engraissement a été de 104,3 jours et le poids moyen initial a été de 27,3 kg. La ration distribuée a été composée de 30 p. 100 de foin de luzerne broyé, 52 p. 100 du maïs grain, 16 p. 100 du TT et 2 p. 100 du CMV. L'abattage a eu lieu lorsque l'âge des agneaux était en moyenne de 168 jours. Les animaux ont fait l'objet de traitements sanitaires et prophylactiques pour prévenir contre l'entérotoxémie, la clavelée, la myopathie et les parasites internes et externes.

\section{Contrôles effectués}

A la naissance, les agneaux ont été identifiés par une boucle d'oreille et leur date de naissance, sexe et type de naissance enregistrés. Ils ont également été pesés à la naissance, puis tous les 15 jours jusqu'au sevrage qui a lieu vers l'âge de 90 jours. Par la suite, ils ont été pesés une fois par mois. Les agneaux mâles sont restés entiers et n'ont pas subi la caudectomie.

Les ovins âgés de plus d'une année ont été tondus manuellement une fois par an vers avril-mai. Le poids de la toison a été enregistré le jour de la tonte.

Durant la période d'engraissement, les contrôles effectués sur les agneaux ont été les suivants :

- triple pesée des agneaux au début et à la fin de la période de l'engraissement ;

- pesées simples tous les 15 jours durant la période d'engraissement ;
- contrôle quotidien de l'alimentation distribuée par lot (pesée des quantités distribuées et refusées) et son ajustement de telle sorte que le refus constituait 10 p. 100 du distribué.

A l'abattage, ont été déterminés le poids vif à l'abattage (après $18 \mathrm{~h}$ de jeûne), le poids de la carcasse chaude (15 min après son obtention), le poids du gras de rognon et la note de l'état d'engraissement à l'aide des modèles photographiques de Colomer-Rocher (13). La note 1 correspond aux carcasses très maigres et la note 5 correspond aux carcasses excessivement grasses.

\section{Variables étudiées}

Les caractères de reproduction étudiés ont été la durée de la gravidité, la fertilité (valeur 1 affectée aux brebis gravides et 0 aux brebis non gravides), la taille et le poids de portée à la naissance (respectivement nombre et poids des agneaux nés), et la taille et le poids de portée au sevrage (respectivement nombre et poids des agneaux présents à 90 jours) des brebis ayant agnelé.

Les caractères de croissance ont été le poids à la naissance, à 30 jours, à 90 jours, à 180 jours, à 360 jours, le gain moyen quotidien (GMQ) entre la naissance et 30 jours, et entre 30 et 90 jours. Les différents poids aux âges types ont été obtenus par interpolation linéaire entre les différentes pesées effectuées.

Les caractères étudiés au cours de la période d'engraissement ont été le niveau d'ingestion, l'indice de consommation, le GMQ à l'engraissement et le coût alimentaire par kilogramme de croît. Les caractéristiques d'abattage et de carcasses étudiées ont été le poids vif à l'abattage, le poids de la carcasse chaude, le rendement économique, le gras de rognon et l'état d'engraissement.

\section{Analyses statistiques}

Les données analysées ont été issues d'un troupeau de race Sardi constitué par l'achat des brebis auprès des éleveurs sélectionneurs du berceau de la race. La base de données a été constituée de 811 performances de reproduction, 1430 poids de toison, 1024 performances de croissance et de viabilité et 154 performances d'engraissement et d'abattage. Toutes les données ont été collectées au DED à l'Inra durant sept campagnes allant de 1993-1994 à 1999-2000. Les moyennes et les écarts-types ont été calculés à l'aide du logiciel SAS (18).

\section{RESULTATS ET DISCUSSION}

\section{Performances de reproduction}

Les brebis du troupeau ont eu un rang d'agnelage qui a varié de 1 à 5 avec une moyenne de 2,7, un âge moyen de 47 mois, un poids moyen à la lutte de $53,4 \mathrm{~kg}$ et un poids moyen à l'agnelage de $59,8 \mathrm{~kg}$. Les moyennes arithmétiques, les écarts-types et les coefficients de variation des caractères de reproduction des brebis de race Sardi sont rapportés dans le tableau I.

La durée de la gravidité des brebis de race Sardi a été en moyenne de 151,4 jours. Cette durée de la gravidité, considérée comme longue, est similaire à celle des brebis de races Boujaâd, Timahdite, Béni Guil et D'man $(1,9,12)$.

Les brebis de race Sardi ont eu un taux de fertilité moyen de 98 p. 100. Cette valeur élevée était identique à celle obtenue chez les brebis de race Boujaâd à la même station (12), mais supérieure à celles rapportées chez les brebis de races Timahdite, Béni Guil, D'man et Sardi qui varient de 74 à 95 p. $100(1,7,10)$.

La taille de portée à la naissance des brebis de race Sardi a varié de 1 à 3 agneaux, avec une moyenne de 1,29 agneau. Les portées simples ont représenté 72 p. 100 , les doubles 27,1 p. 100 et 


\section{Tableau I}

Moyennes arithmétiques, coefficients de variation, minimums et maximums des performances de reproduction des brebis de race Sardi

\begin{tabular}{|c|c|c|c|c|c|}
\hline Caractère & Nb. & $\begin{array}{l}\text { Moy. } \\
\text { arithm. }\end{array}$ & $\begin{array}{l}\text { Coeff. } \\
\text { var. }(\%)\end{array}$ & Min. & Max. \\
\hline $\begin{array}{l}\text { Durée de } \\
\text { la gravidité (j) }\end{array}$ & 793 & 151,4 & 1,2 & 145 & 155 \\
\hline Fertilité (\%) & 811 & 98 & 14,3 & 0 & 100 \\
\hline $\begin{array}{l}\text { Taille de la portée } \\
\text { à la naissance }\end{array}$ & 793 & 1,29 & 31,8 & 1,00 & 3,00 \\
\hline $\begin{array}{l}\text { Taille de la portée } \\
\text { au sevrage }\end{array}$ & 793 & 1,25 & 32,8 & 1,00 & 3,00 \\
\hline $\begin{array}{l}\text { Poids de la portée } \\
\text { à la naissance (kg) }\end{array}$ & 793 & 5,30 & 28,1 & 3,00 & 11,3 \\
\hline $\begin{array}{l}\text { Poids de la portée } \\
\text { au sevrage (kg) }\end{array}$ & 793 & 27,9 & 26,5 & 14,3 & 64,5 \\
\hline
\end{tabular}

les triples 0,9 p. 100. La taille de portée des brebis de race Sardi a été similaire à celle réalisée par les brebis de race Boujaâd à la même station (12). Cependant, elle a été supérieure aux tailles de portée à la naissance comprises entre 1,05 et 1,21 obtenues chez les brebis de races Timahdite et Béni Guil, ainsi que chez les brebis de race Sardi élevées dans des conditions différentes. Par ailleurs, les brebis de race Sardi ont sevré 1,24 agneau. Ce résultat a été supérieur à ceux enregistrés chez les brebis des races Timahdite et Béni Guil qui varient en moyenne de 0,99 à 1,19 agneau (4, $7,9,10)$, identique à celui des brebis de race Boujaâd élevées dans des conditions similaires (12), mais inférieur à celui des brebis de races prolifiques D'man et DS $(2,9)$.

Le poids de portée à la naissance des brebis de race Sardi a été en moyenne de $5,30 \mathrm{~kg}$. Il a été plus élevé que celui des brebis de race Timahdite et Béni Guil qui varient de 3,60 à 4,18 kg, mais identique à celui des brebis Boujaâd (12). A 90 jours, les brebis ont sevré des portées dont le poids a été en moyenne de $27,9 \mathrm{~kg}$. Ce poids a été identique à celui des brebis de race Boujaâd, mais de loin supérieur à tous les poids de portée au sevrage obtenus sur les races Timahdite, Béni Guil et Sardi qui oscillent entre 15,1 et 23,5 kg (1, 4, 7, 10).

Les performances de reproduction réalisées par les brebis de race Sardi en station ont montré que les résultats obtenus dans le passé étaient sous-estimés et que la race avait un bon potentiel génétique qui lui permettait de réaliser des productivités élevées. La différence entre les résultats de la présente étude et ceux des études précédentes peut être attribuée au mode de conduite surtout alimentaire des brebis durant les phases physiologiques critiques. Dans la présente étude, les animaux ont été conduits de façon intensive avec une alimentation à l'auge qui a couvert leurs besoins d'entretien et de production. Dans les autres études, l'alimentation des animaux était souvent insuffisante et déséquilibrée car essentiellement basée sur les parcours.

\section{Production de laine}

Le poids de la toison des ovins de race Sardi tondus une fois par an a été en moyenne de $2,03 \mathrm{~kg}$. Il a été de $1,92 \mathrm{~kg}$ chez les brebis et de $2,89 \mathrm{~kg}$ chez les béliers (tableau II). La valeur moyenne a été comparable aux poids obtenus chez les ovins de races locales Béni Guil, Timahdite et Béni Ahsen qui sont en moyenne de $2 \mathrm{~kg}$ (1), mais inférieure à la moyenne de $3,47 \mathrm{~kg}$ enregistrée chez les ovins de race Boujaâd (12).

\section{Tableau II}

Moyennes arithmétiques, coefficients de variation, minimums et maximums des poids de la toison des ovins de race Sardi

\begin{tabular}{lrcccc} 
Catégorie & Nb. & $\begin{array}{c}\text { Moy. } \\
\text { arithm. } \\
(\mathbf{k g})\end{array}$ & $\begin{array}{c}\text { Coeff. } \\
\text { var.(\%) }\end{array}$ & $\begin{array}{c}\text { Min. } \\
(\mathbf{k g})\end{array}$ & $\begin{array}{c}\text { Max. } \\
(\mathbf{k g})\end{array}$ \\
\hline Béliers & 158 & 2,89 & 30,3 & 0,80 & 5,90 \\
Brebis & 1272 & 1,92 & 26,9 & 0,70 & 5,40 \\
Ensemble & 1430 & 2,03 & 23,0 & 0,70 & 5,90
\end{tabular}

\section{Performances de croissance et de viabilité des agneaux}

Les performances de croissance et de viabilité des agneaux sont rapportées dans le tableau III. Les poids à la naissance, à 30 jours, à 90 jours, à 6 mois et à 1 an des agneaux de race Sardi ont été respectivement de $4,10 \mathrm{~kg}, 10,9 \mathrm{~kg}, 22,5 \mathrm{~kg}, 35,7 \mathrm{~kg}$ et $58,6 \mathrm{~kg}$. Leurs vitesses de croissance entre la naissance et 30 jours et entre 30 et 90 jours ont été respectivement de $224 \mathrm{~g} / \mathrm{j}$ et $194 \mathrm{~g} / \mathrm{j}$. Les performances de croissance ont différé selon le sexe de l'agneau. Les mâles ont pesé 4,22 kg à la naissance, $11,3 \mathrm{~kg}$ à 30 jours, 23,6 kg à 90 jours, $38,5 \mathrm{~kg}$ à 6 mois et $64,1 \mathrm{~kg}$ à 1 an, alors que les femelles ont pesé respectivement $4 \mathrm{~kg}, 10,5 \mathrm{~kg}, 21,0 \mathrm{~kg}, 33,3 \mathrm{~kg}$ et $53,0 \mathrm{~kg}$ (figure 1). La vitesse de croissance entre la naissance et 30 jours a été de $234 \mathrm{~g} / \mathrm{j}$ chez les agneaux et de $215 \mathrm{~g} / \mathrm{j}$ chez les agnelles, celle entre 30 et 90 jours a été de $205 \mathrm{~g} / \mathrm{j}$ chez les agneaux et de $184 \mathrm{~g} / \mathrm{j}$ chez les agnelles. Les poids vifs et les vitesses de croissance obtenus dans la présente étude ont été plus élevés à tous les âges que toutes les valeurs obtenues jusqu'à présent sur la race Sardi et les autres races locales Timahdite, Béni Guil et Boujaâd, témoignant du potentiel génétique élevé pour la croissance des agneaux de race Sardi. En effet, les poids rapportés chez les races Sardi, Timahdite, Béni Guil, DS et Boujaâd oscillent entre 3 et $3,89 \mathrm{~kg}$ à la naissance, entre 6,9 et $9,8 \mathrm{~kg}$ à 30 jours, entre 15 et $21,2 \mathrm{~kg}$ à 90 jours. Les vitesses de croissance varient en moyenne de 116 à $215 \mathrm{~g} / \mathrm{j}$ entre la naissance et 30 jours et de 127 à $190 \mathrm{~g} / \mathrm{j}$ entre 30 et 90 jours $(2,3,5,8,11,12)$.

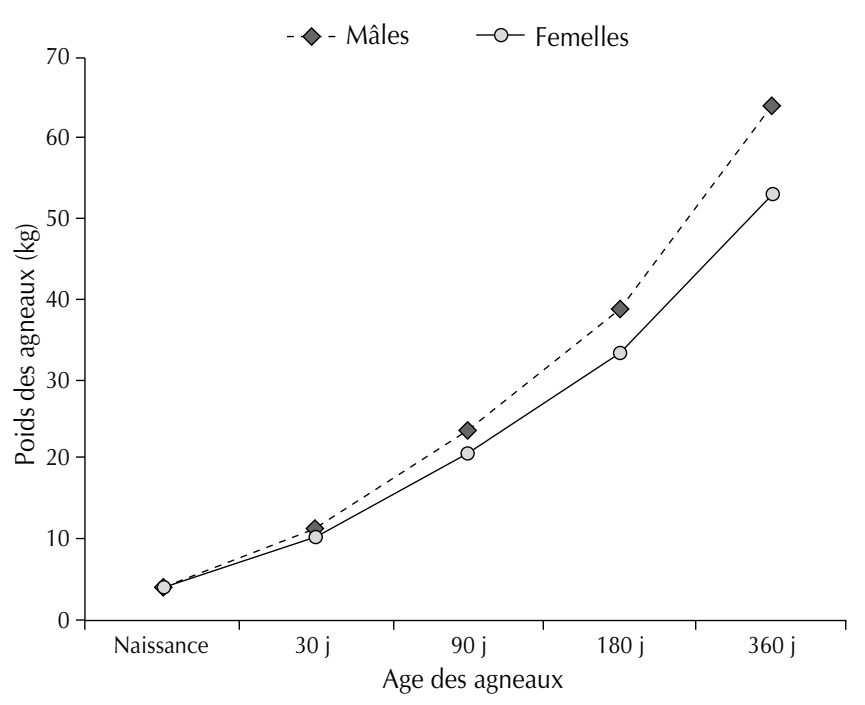

Figure 1 : évolution des poids des agneaux de race Sardi selon l'âge. 
Tableau III

Moyennes arithmétiques, coefficients de variation, minimums et maximums des performances de croissance et de viabilité des agneaux de race Sardi

\begin{tabular}{|c|c|c|c|c|c|c|}
\hline Sexe & Caractère & Nb. & Moy. arithm. & Coeff. var. (\%) & Min. & Max. \\
\hline \multirow[t]{8}{*}{ Mâle } & Poids à la naissance (kg) & 477 & 4,22 & 14,9 & 2,00 & 6,00 \\
\hline & Poids à 30 j (kg) & 443 & 11,3 & 15,9 & 7,20 & 15,7 \\
\hline & Poids à 90 j (kg) & 438 & 23,6 & 16,1 & 12,3 & 33,7 \\
\hline & Poids à 180 j (kg) & 44 & 38,5 & 14,8 & 27,3 & 48,3 \\
\hline & Poids à 360 j (kg) & 44 & 64,1 & 11,7 & 46,8 & 80,3 \\
\hline & GMQ 0-30 j (g) & 443 & 234 & 20,9 & 124 & 370 \\
\hline & GMQ 30-90 j (g) & 438 & 205 & 19,5 & 75 & 332 \\
\hline & Viabilité $0-90$ j (\%) & 477 & 93 & 26,9 & 0 & 100 \\
\hline \multirow[t]{8}{*}{ Femelle } & Poids à la naissance (kg) & 547 & 4,00 & 14,7 & 2,00 & 5,70 \\
\hline & Poids à 30 j (kg) & 513 & 10,5 & 14,3 & 5,8 & 14,5 \\
\hline & Poids à 90 j (kg) & 509 & 21,0 & 12,4 & 11,3 & 29,2 \\
\hline & Poids à 180 j (kg) & 51 & 33,3 & 11,7 & 24,1 & 42,9 \\
\hline & Poids à 360 j (kg) & 44 & 53,0 & 9,8 & 45,2 & 66,5 \\
\hline & GMQ 0-30 j (g) & 513 & 215 & 17,7 & 70 & 325 \\
\hline & GMQ 30-90 j (g) & 509 & 184 & 13,6 & 92 & 273 \\
\hline & Viabilité 0-90 j (\%) & 547 & 93 & 26,9 & 0 & 100 \\
\hline \multirow[t]{8}{*}{ Ensemble } & Poids à la naissance (kg) & 1024 & 4,10 & 12,2 & 2,00 & 6,00 \\
\hline & Poids à 30 j (kg) & 956 & 10,9 & 11,0 & 5,8 & 15,7 \\
\hline & Poids à 90 j (kg) & 947 & 22,5 & 10,7 & 11,3 & 33,7 \\
\hline & Poids à 180 j (kg) & 95 & 35,7 & 12,0 & 24,1 & 48,3 \\
\hline & Poids à 360 j (kg) & 88 & 58,6 & 10,4 & 45,2 & 80,3 \\
\hline & GMQ 0-30 j (g) & 956 & 224 & 15,2 & 70 & 370 \\
\hline & GMQ 30-90 j (g) & 947 & 194 & 13,9 & 75 & 332 \\
\hline & Viabilité 0-90 j (\%) & 1024 & 93 & 26,9 & 0 & 100 \\
\hline
\end{tabular}

Le taux de viabilité entre la naissance et 90 jours des agneaux de race Sardi a été de 93 p. 100 (tableau III). Il a été identique à celui enregistré chez les agneaux de race Boujaâd (12), mais supérieur aux taux de viabilité obtenus chez les agneaux des races Timahdite, Béni Guil, DS et D'man qui varient de 75 à 93 p. $100(1,2,3,5,9$, 15). Il est à noter que 76,7 p. 100 des mortalités entre la naissance et le sevrage ont eu lieu entre la naissance et 10 jours, 10 p. 100 entre 10 et 30 jours et 13,3 p. 100 entre 30 et 90 jours. Cette distribution des mortalités selon l'âge est conforme à celles rapportées par Prud'hon et coll. (16) et Ricordeau et coll. (17) qui montrent que 50 à 80 p. 100 de l'ensemble des mortalités pré-sevrage se produisent durant la première semaine de vie des agneaux.

Les différences entre les performances de croissance et de viabilité des agneaux de race Sardi dans la présente étude et celles obtenues aussi bien chez la race Sardi que chez les autres races locales dans les études antérieures peuvent être attribuées en grande partie à l'alimentation des brebis et des agneaux à travers son effet favorable sur la production laitière des mères et sur la vigueur des produits.

\section{Performances d'engraissement et caractéristiques de carcasses}

Les performances d'engraissement et des caractéristiques de carcasses des agneaux mâles de race Sardi sont présentées dans le tableau IV. Les agneaux de race Sardi ont réalisé un GMQ à l'engraissement de $283 \mathrm{~g} / \mathrm{j}$. Ce gain a été similaire à celui obtenu chez les agneaux de race Boujaâd (12), mais plus élevé que les GMQ variant entre 142 et $215 \mathrm{~g} / \mathrm{j}$ réalisés par les agneaux des races Timahdite, Béni Guil, Sardi et D'man $(7,9,11)$.

Durant la période d'engraissement, le niveau d'ingestion a été en moyenne de $1,36 \mathrm{~kg}$ de matière sèche $(\mathrm{MS}) / \mathrm{animal} / \mathrm{j}$ et de $78 \mathrm{~g}$ de
$\mathrm{MS} / \mathrm{kg} \mathrm{P}^{0,75} / \mathrm{j}$. Cette performance, comparable à celle des agneaux de race Boujaâd, a été plus faible que les niveaux de 1,38 à 1,51 kg de MS/animal/j et de 104 à $108 \mathrm{~g}$ de MS/kg $\mathrm{P}^{0,75} / \mathrm{j}$ enregistrés chez les agneaux issus du croisement entre les béliers de race Ile de France et les brebis de races Sardi, Béni Guil et Timahdite (6).

L'indice de consommation a été en moyenne de 4,87 kg de MS d'aliments/kg de gain de poids et de 4,32 unités fourragères viande $(\mathrm{UFV}) / \mathrm{kg}$ de gain de poids. Il a été plus faible que les valeurs de $4,70 \mathrm{~kg}$ de MS d'aliments $/ \mathrm{kg}$ de gain de poids et de 4,17 UFV $/ \mathrm{kg}$ de gain de poids obtenues chez les agneaux de race Boujaâd (12), et que les valeurs de 5,7 à 8,4 kg de MS d'aliments/kg de gain de poids enregistrées chez les agneaux de races Sardi, Béni Guil et Timahdite $(6,9)$.

A l'abattage, le poids vif des agneaux de race Sardi a été de $45,5 \mathrm{~kg}$ et leur poids de carcasse chaude de $23,2 \mathrm{~kg}$. Ces poids ont été plus élevés que ceux rapportés par Boujenane et coll. (6) chez les agneaux des races Sardi, Béni Guil et Timahdite, conduites en races pures ou en croisement avec les béliers des races à viande étrangères, qui sont en moyenne de 32,2 et 15,6 kg.

Le rendement en carcasse des agneaux de race Sardi a été en moyenne de 50,9 p. 100. Il a été plus élevé que le rendement moyen trouvé chez les agneaux de races D'man, Sardi, Béni Guil et Timahdite qui est en moyenne de 48 p. $100(6,9)$, et chez les agneaux issus du croisement entre les races locales et les races à viande qui est de 48,7 p. 100 (6).

La note d'état d'engraissement des carcasses des agneaux de race Sardi a été en moyenne de 3,91. Ce score correspondait à la catégorie grasse et était analogue à ceux observés chez les races Sardi, Béni Guil, Timahdite et Boujaâd qui varient de 3,6 à 4,4 $(6,12)$. 


\section{Tableau IV}

Moyennes arithmétiques, coefficients de variation, minimums et maximums des performances d'engraissement et caractéristiques de carcasse des agneaux mâles de race Sardi

\begin{tabular}{|c|c|c|c|c|c|}
\hline Caractère & $\mathrm{Nb.}$ & $\begin{array}{l}\text { Moy. } \\
\text { arithm. }\end{array}$ & $\begin{array}{l}\text { Coeff. } \\
\text { var. }(\%)\end{array}$ & Min. & Max. \\
\hline $\begin{array}{l}\text { GMQ à } \\
\text { l'engraissement (g) }\end{array}$ & 154 & 283 & 12,7 & 203 & 396 \\
\hline $\begin{array}{l}\text { Niveau d'ingestion } \\
\text { (kg MS/animal/j) }\end{array}$ & 154 & 1,36 & 11,8 & 1,24 & 1,60 \\
\hline $\begin{array}{l}\text { Niveau d'ingestion } \\
\left.\text { (g MS/kg } \mathrm{P}^{0,75} / \mathrm{j}\right)\end{array}$ & 154 & 77,8 & 11,0 & 68,1 & 97,9 \\
\hline $\begin{array}{l}\text { Indice de consommation } \\
\text { (kg MS/kg de } \\
\text { gain de poids) }\end{array}$ & 154 & 4,87 & 13,1 & 3,80 & 6,88 \\
\hline Poids vif à l'abattage (kg) & 154 & 45,5 & 8,3 & 36,5 & 57,1 \\
\hline $\begin{array}{l}\text { Poids de carcasse } \\
\text { chaude (kg) }\end{array}$ & 154 & 23,2 & 9,9 & 17,8 & 30,8 \\
\hline $\begin{array}{l}\text { Rendement } \\
\text { économique (\%) }\end{array}$ & 154 & 50,9 & 4,9 & 45,2 & 55,1 \\
\hline $\begin{array}{l}\text { Note de l'état } \\
\text { d'engraissement }\end{array}$ & 154 & 3,91 & 38,4 & 2 & 5 \\
\hline $\begin{array}{l}\text { Poids du gras } \\
\text { rognon }(\mathrm{g})\end{array}$ & 154 & 295 & 29,5 & 120 & 640 \\
\hline $\begin{array}{l}\text { Coût alimentaire } \\
\left(\mathrm{DH}^{*} / \mathrm{kg} \text { du gain du poids }\right)\end{array}$ & 154 & 10,1 & 15,0 & 8,44 & 14,5 \\
\hline
\end{tabular}

* Dirhams

Le poids du gras de rognon des agneaux de race Sardi a été de 295 g. Il a été identique à celui des agneaux de race Boujaâd, mais plus élevé que ceux des agneaux de races D'man, Sardi, Béni Guil et Timahdite ou des agneaux issus du croisement entre les races locales et les races à viande qui varient en moyenne de 99 à $260 \mathrm{~g}$ $(6,9)$.

Le coût alimentaire de l'engraissement des agneaux Sardi a été de 10,1 dirhams (DH) par kilogramme du gain de poids vif et de 20,2 DH par kilogramme du gain de poids de carcasse (1€ $\approx 10,9 \mathrm{DH})$. Ces coûts ont été inférieurs à ceux trouvés chez les races Sardi et D'man par El Aouni (14) qui sont respectivement de 21,2 DH et 23,2 DH par kilogramme du gain du poids de carcasse. Cependant, ils ont été supérieurs aux coûts obtenus chez la race Boujaâd de 9,6 DH par kilogramme du gain du poids vif et de 19,2 DH par kilogramme du gain du poids de carcasse (12).

\section{CONCLUSION}

Les performances des ovins de race Sardi ont été élevées et les résultats rapportés dans les travaux réalisés dans le passé ne reflètent pas le vrai potentiel de la race, car ils ont été obtenus dans des conditions de conduite en mode extensif. Par conséquent, des plans d'alimentation adéquats sont recommandés afin d'atteindre des performances meilleures et d'aboutir à des productivités plus importantes et donc à une production de viande élevée.

\section{Remerciements}

Les auteurs adressent leurs vifs remerciements à M. L. Haounou, technicien au domaine expérimental Déroua, pour sa collaboration technique.

\section{BIBLIOGRAPHIE}

1. BOUJENANE I., 1999. Les ressources génétiques ovines au Maroc. Rabat, Maroc, Actes Editions, 136 p.

2. BOUJENANE I., 2002. Development of the DS synthetic breed of sheep in Morocco: ewe reproduction and lamb preweaning growth and survival. Small Ruminant Res., 45: 61-66.

3. BOUJENANE I., BRADFORD G.E., BERGER Y.M., CHIKHI A., 1991. Genetic and environmental effects on growth to 1 year and viability of lambs from crossbreeding study of D'man and Sardi breeds. J. Anim. Sci., 69: 3989-3998.

4. BOUJENANE I., MHARCHI A., 1992. Estimation des paramètres génétiques et phénotypiques des performances de reproduction des brebis de race Beni Guil. Actes Inst. agron. vét. (Maroc), 12 : 5-13.

5. BOUJENANE I., MHARCHI A., 1992. Estimation des paramètres génétiques et phénotypiques des performances de croissance et de viabilité des agneaux de race Beni Guil. Actes Inst. agron. vét. (Maroc), 12 : 15-22.

6. BOUJENANE I., BERRADA D., MIHI S., JAMAI M., 1996. Performances d'engraissement et caractéristiques des carcasses des agneaux issus du croisement des brebis de races Timahdite, Sardi et Beni Guil avec des béliers de races à viande. Actes Inst. agron. vét. (Maroc), 16 : 29-38.

7. BOUJENANE I., BERRADA D., MIHI S., JAMAI M., 1998. Reproductive performances of ewes and preweaning growth of lambs from three native Moroccan breeds mated to rams from Moroccan and improved breeds. Small Ruminant Res., 27: 203-208.

8. BOUJENANE I., M'ZIAN S., SADIK M., 2001. Estimation des paramètres génétiques et phénotypiques de la croissance des ovins de race Sardi. Actes Inst. agron. vét., (Maroc), 21 : 177-183.

9. BOUJENANE I., ROUDIES N., BENMIRA A., EL IDRISSI Z., EI AOUNI M., 2003. On-station assessment of performance of the DS synthetic and parental sheep breeds, D'man and Sardi. Small Ruminant Res., 49: 125-133.

10. BOURFIA M., TOUCHBERRY R.W., 1993. Diallel cross of three Moroccan breeds of sheep. II. Reproductive performance and productivity of pure breeds ewes. J. Anim. Sci., 71: 882-887.

11. BOURFIA M., TOUCHBERRY R.W., 1993. Diallel cross of three Moroccan breeds of sheep. I. Lamb growth and carcass traits. J. Anim. Sci., 71: 870-881.

12. CHIKHI A., BOUJENANE I., 2003. Performances de reproduction et de production des ovins de race Boujaâd au Maroc. Revue Elev. Méd. vét. Pays trop., 56 : 83-88.

13. COLOMER-ROCHER F., 1986. Méthode normalisée pour l'étude des caractères quantitatifs et qualitatifs des carcasses ovines, produites dans le bassin méditerranéen, en fonction des systèmes de production. Les carcasses d'agneaux et de chevreaux méditerranéens. Saragosse, Espagne, Ciheam, 22 p. (Rapport eur. 11479 Fr.)

14. EL AOUNI M., 1990. Comparaison des performances laitières des brebis et performances d'engraissement et de carcasses des agneaux des races DS, D'man et Sardi. Mémoire $3^{\mathrm{e}}$ cycle Agronomie, Institut agronomique et vétérinaire Hassan II, Rabat, Maroc.

15. EL FADILI M., MICHAUX C., BOULANOUAR B., LEROY P.L., 2000 Environmental and genetic effects on growth in Timahdite and crossbred lambs in Morocco. Revue Elev. Méd. vét. Pays trop., 53: 75-83.

16. PRUD'HON M., DENOY I., DESVIGNES A., 1968. Etude des résultats de six années d'élevage des brebis Mérinos d'Arles du domaine de Merle. III. La mortalité des agneaux. Ann. Zootech., 17 : 159-168.

17. RICORDEAU G., TCHAMITCHIAN L., LEFEVRE C., BRUNEL J.C., 1977. Amélioration de la productivité des brebis Berrichonnes du Cher (BC) par croisement. IV. Durée de la gravidité et viabilité des agneaux Berrichon, Romanov et croisés F1, F2, et F3. Ann. Génét. Sél. Anim., 9 : 219-239.

18. SAS/STAT, 1996. User's guide, vers. 6.12. Cary, NC, USA, SAS Institute.

Reçu le 09.02.2004, accepté le 28.05.2004 


\section{Summary}

Chikhi A., Boujenane I. Characterization of the Sardi Sheep Breed in Morocco

A total of 811 reproduction records, 1430 fleece weights, 1024 growth and viability records, and 154 fattening and carcass quality records of Sardi sheep were analyzed. Data were collected over seven periods from 1993-1994 to 19992000 at Deroua Experimental Station of INRA. Average gestation length of Sardi ewes was 151 days, fertility rate was $98 \%$, litter sizes at birth and at weaning (90 days) were 1.29 and 1.25 lambs, respectively, and litter weights at birth and at weaning were 5.30 and $27.9 \mathrm{~kg}$, respectively. Lamb weight was $4.10 \mathrm{~kg}$ at birth, $10.9 \mathrm{~kg}$ at 30 days and $22.5 \mathrm{~kg}$ at 90 days. Average daily gain was $224 \mathrm{~g}$ from birth to 30 days and $194 \mathrm{~g}$ from 30 to 90 days. The mortality rate of lambs from birth to 90 days was $7 \%$. After an average fattening period of 66.4 days, the average daily gain of Sardi lambs born single was $283 \mathrm{~g}$ and feed efficiency averaged $4.87 \mathrm{~kg}$ of feed per kilogram of weight gained. Slaughtered at 168 days of age on average, slaughter weight of lambs averaged $45.5 \mathrm{~kg}$, hot carcass weight $23.2 \mathrm{~kg}$, dressing percentage $50.9 \%$, and weight of kidney fat $295 \mathrm{~g}$. It was concluded that the Sardi breed had high potential that could be developed to increase meat production in Morocco.

Key words: Sardi sheep - Animal performance - Reproductive performance - Body weight - Wool - Carcass - Morocco.

\section{Resumen}

Chikhi A., Boujenane I. Caracterización zootécnica de los ovinos de raza Sardi en Marruecos

Se analizaron un total de 811 rendimientos de reproducción, 1430 pesos de vellón, 1024 rendimientos de crecimiento y viabilidad y 154 rendimientos de engorde y de sacrificio de los ovinos de raza Sardi. Estos rendimientos se recolectaron durante siete campañas entre 1993-1994 y 1999-2000 en el dominio experimental de Deroua en el Instituto nacional de investigación agronómica. El análisis de los datos demostró que las ovejas tuvieron una duración de gravidez de 151 días, una tasa de fertilidad de $98 \%$, tamaños respectivos de camadas al nacimiento y al destete (90 días) de 1,29 y 1,25 y pesos respectivos de la camada al nacimiento y al destete 5,30 y $27,9 \mathrm{~kg}$. Los corderos pesaron 4,10 kg al nacimiento, 10,9 kg a los 30 días y $22,5 \mathrm{~kg}$ a los 90 días. La velocidad de crecimiento promedio fue de $224 \mathrm{~g} / \mathrm{d}$ del nacimiento a los 30 días y de $194 \mathrm{~g} / \mathrm{d}$ entre el día 30 y el 90. La tasa de mortalidad de los corderos entre el nacimiento y el día 90 fue de $7 \%$. Después de un engorde post destete de una duración promedio de 66,4 días, los corderos de partos simples tuvieron un GMQ de $283 \mathrm{~g} / \mathrm{d}$ y un índice de consumo de $4,87 \mathrm{~kg}$ de alimento/kg de ganancia de peso. Sacrificados a una edad promedio de 168 días, presentaron un peso vivo al sacrificio de $45,5 \mathrm{~kg}$, un peso de carcasa caliente de $23,2 \mathrm{~kg}$, un rendimiento económico de 50,9\% y un peso de grasa de riñón de 295 g. Se concluye que la raza Sardi tiene un buen potencial, cuya explotación es posible con el fin de aumentar la producción de carne en Marruecos.

Palabras clave: Ovino Sardi - Desempeno animal - Reproductividad - Peso corporal - Lana - Canal animal - Marruecos. 